\title{
Part 1: CT characterisation of pancreatic neoplasms: a pictorial essay
}

\author{
Angela Galvin • Tom Sutherland • Andrew F. Little
}

Received: 11 January 2011 /Revised: 28 February 2011 / Accepted: 4 May 2011 / Published online: 18 May 2011

(C) European Society of Radiology 2011

\begin{abstract}
The pancreas is a site of origin of a diverse range of benign and malignant tumours, and these are frequently detected, diagnosed and staged with computed tomography (CT). Knowledge of the typical appearance of these neoplasms as well as the features of locoregional invasion is fundamental for all general and abdominal radiologists. This pictorial essay aims to outline the characteristic CT appearances of the spectrum of pancreatic neoplasms, as well as important demographic and clinical information that aids diagnosis. The second article in this series addresses common mimics of pancreatic neoplasia.
\end{abstract}

Keywords Pancreatic neoplasms - Computed tomography CT

\section{Imaging technique}

Imaging of the pancreas is typically achieved with dual- or triple-phase acquisitions. Imaging is performed in the noncontrast, arterial and delayed portal venous phases using rapid contrast agent administration at $3-5 \mathrm{ml} / \mathrm{s}$, preferably delivered via a wide bore cannula. High-density oral contrast agent interferes with the delineation of vessels and it can also make appreciation of an arterially enhancing lesion more difficult. Oral administration of $500-700 \mathrm{ml}$ water approximately $30 \mathrm{~min}$ before imaging time is a safe and effective way to achieve distension of the upper gastrointestinal tract [1]. Water acts as a negative contrast

A. Galvin $\cdot$ T. Sutherland $(\bowtie) \cdot$ A. F. Little

Medical Imaging Department, St Vincent's Hospital,

41 Victoria Pde,

3065 Fitzroy, Australia

e-mail: Tom.sutherland@svhm.org.au agent, differentiating the duodenum from the adjacent pancreatic head. Positive oral contrast agent may also be administered as part of the imaging preparation $60 \mathrm{~min}$ before imaging if lower abdominal and pelvic delineation of bowel loops from adjacent structures is required. Postresection imaging may require a positive contrast agent to differentiate bowel loops from fluid collections and to help identify anastomotic leaks.

\section{Pancreatic ductal adenocarcinoma}

Pancreatic ductal adenocarcinoma accounts for $90 \%$ of all pancreatic neoplasms. Most of the patients are over 60 years of age at diagnosis. It is a common malignancy in both men and women and has a low 5-year survival rate [2], reflecting the predominance of late diagnosis and low resection rates at presentation. These tumours grow rapidly and metastasise early and only $10-15 \%$ of patients have tumours that are potentially resectable at diagnosis [3]. Presenting symptoms can include jaundice, weight loss and abdominal pain. Risk factors include chronic pancreatitis, diabetes mellitus and hereditary cancer syndromes. Given the significant morbidity and mortality of this malignancy, early detection and accurate determination of resectability are of vital importance.

Ductal adenocarcinomas occur in the pancreatic head in $78 \%$, with $11 \%$ involving the body and $11 \%$ in the tail [2]. The earliest consistent imaging finding is dilatation of the main pancreatic duct [4]. Sixty percent of small adenocarcinomas show duct dilatation without a visible mass [4]. The combination of a dilated pancreatic duct with a dilated common bile duct ('double duct sign') is highly suspicious for a pancreatic head malignancy. The tumour itself tends to be a hypodense mass that distorts the contour of the gland 
(Fig. 1), although it may be isodense to pancreatic parenchyma on a unenhanced study. Tumour margins are best defined on the arterial phase, with maximum enhancement of the normal pancreatic parenchyma compared with the relatively poor or non-existent enhancement of the tumour [5]. Adenocarcinomas may be cystic (Fig. 2). Postprocessing displays such as maximum intensity projection (MIP) can help define arterial structures. Coronal oblique display allows optimal depiction of the portal vein [6]. Other important diagnostic features include atrophy of the pancreatic tissue proximal to the tumour and a local fibrotic tissue response [7].

The portal venous phase of imaging more clearly defines pancreatic duct dilatation, peripancreatic tissue involvement and distant metastases [8]. Features that determine nonresectability include extrapancreatic invasion of vessels, perineural invasion, adjacent thrombosis of venous structures, such as the splenic vein, splenoportal confluence or the extrahepatic main portal vein, regional lymph node disease as well as omental and distant metastases. Lymphatic invasion may be seen as reticular soft tissue densities or cuff surrounding the extrapancreatic vessels [9], while perineural invasion appears as soft tissue passing in continuity from the primary tumour along the path of a known neural plexus and is most frequently seen around the superior mesenteric artery [10]. Limited invasion of the superior mesenteric vein (SMV) without thrombosis may not rule out resection and some institutions will resect tumours with isolated involvement of the proximal portal vein [6]. Tumours with splenic vessel involvement may be resected with additional en bloc splenectomy. Venous invasion is often described in terms of circumferential contiguity of the

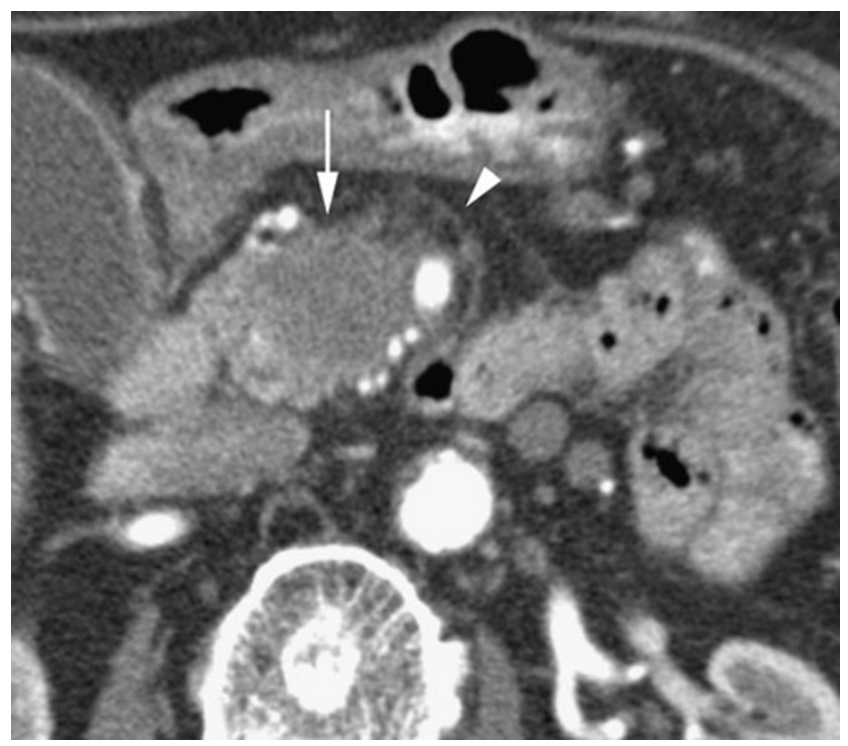

Fig. 1 Arterial phase CT in a 78-year-old woman with a hypodense adenocarcinoma (long arrow) in the pancreatic head and invasion of peripancreatic fat (arrowhead)

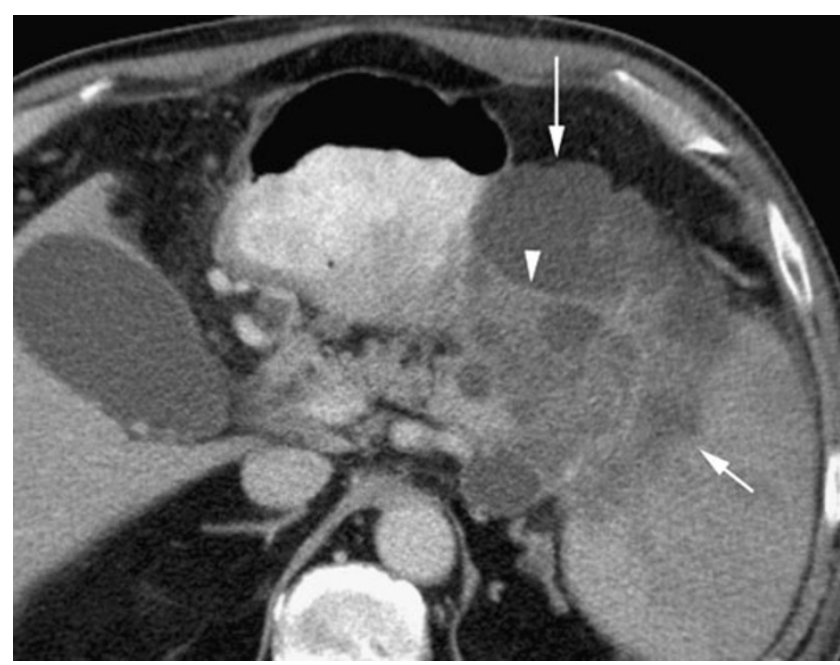

Fig. 2 Mixed cystic and solid adenocarcinoma (long arrow) with irregular soft tissue elements (arrowhead) with invasion of the spleen (short arrow)

tumour with the vein. If more than $50 \%\left(180^{\circ}\right)$ of the SMV is contacted then the tumour will not be resectable [11]. Identifying arterial involvement can be difficult, especially in the presence of atherosclerotic disease. Direct visualisation of abrupt change in vessel calibre and associated soft tissue must be considered in determining arterial invasion [11]. Superior mesenteric artery (SMA) involvement will render the tumour non-resectable (Fig. 3).

The following computed tomography (CT) signs of vascular invasion all have specificities of over $90 \%$ for

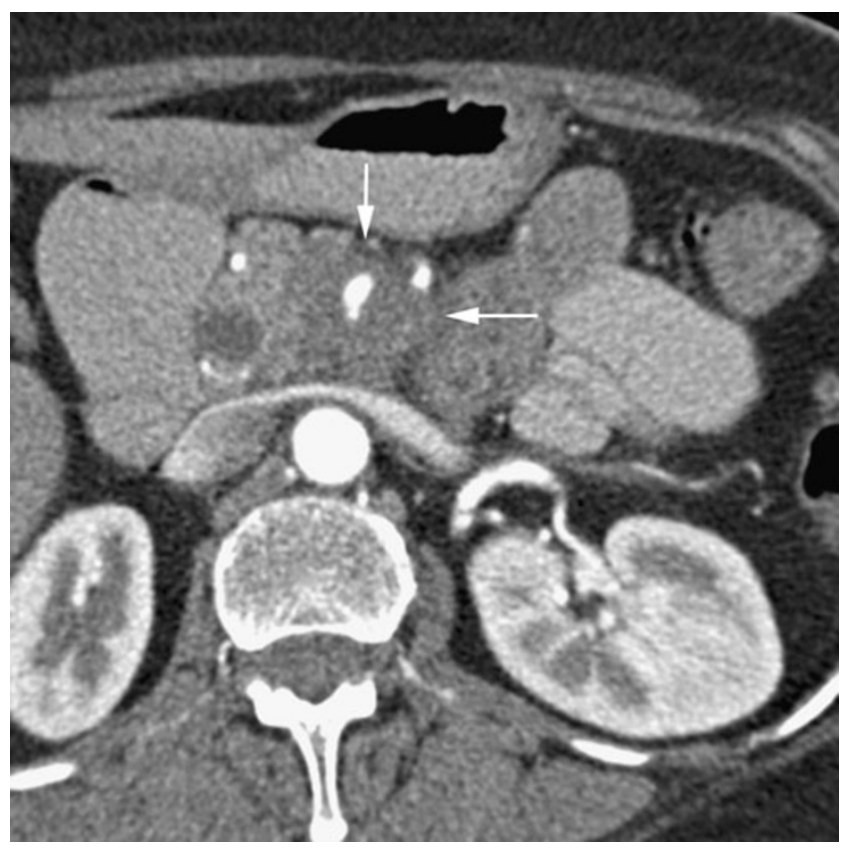

Fig. 3 Arterial phase CT showing a hypodense adenocarcinoma (arrows) in a 64-year-old woman encasing the superior mesenteric artery 
both arterial and venous invasion. Combinations of these signs provide even greater specificity [12-14]: (1) vessel embedded in tumour; (2) tumour contact with over $180^{\circ}$ of vessel circumference; (3) vascular irregularity; (4) vascular stenosis or thrombosis.

A meta-analysis demonstrated that CT had a sensitivity of $91 \%$ and a specificity of $85 \%$ for diagnosing pancreatic adenocarcinoma outperforming magnetic resonance imaging (MRI) [15]. CT has a sensitivity of $81 \%$ for determining resectability and a specificity of $82 \%$ [15]. Other studies show a negative predictive value for resectability of $100 \%$ and a positive predictive value of $89 \%$ with no significant difference between systems of different generations [16]. Differentiating post-operative inflammatory changes from tumour recurrence can also be challenging and serial imaging is frequently required [17].

\section{Cystic neoplasms}

Cystic pancreatic neoplasms range from benign to malignant, and although they may have diagnostic features on CT, often other techniques - such as ultrasound and MRI — are required for further evaluation. It is important to differentiate cystic neoplasm from non-neoplastic pancreatic cysts, which is discussed further in Part 2 of this series. Overall, cystic neoplasms of the pancreas are relatively uncommon, accounting for only $1 \%$ of all primary pancreatic neoplasms [18].

\section{Serous cystic neoplasms}

Previously referred to as serous cystadenomas, these tumours are further divided into microcystic and macrocystic (oligocystic) subgroups based on macroscopic variation.

\section{Microcystic adenoma}

Microcystic adenomas are rare benign pancreatic tumours that are more common in females and tend to occur in the 7th decade of life - therefore termed the 'grandmother' tumour [18]. Despite the predilection for females, cases do occur in males. This can be helpful in differentiating these lesions from mucinous cystic neoplasms, as these almost never occur in males [19]. Microcystic adenomas may be discovered incidentally or present with non-specific abdominal symptoms such as nausea or abdominal pain.

The classic appearance of a microcystic adenoma is of a large hypodense mass composed of a conglomerate of small cysts, each not more than $2 \mathrm{~cm}$ in size. There are multiple thin septations that are highly vascular and therefore enhance on post-contrast-enhanced imaging (Fig. 4) [19]. This results in a 'honeycombed' appearance. The supplying vessels to the mass are frequently enlarged [19]. Another common feature is a central hyperdense scar that may contain calcifications (Fig. 5) [18]. The density of the lesion can vary with internal protein content from water density to muscle equivalent density [20]. The hypervascularity of the septae can also result in internal haemorrhage.

Microcystic adenomas occur slightly more commonly in the pancreatic head and can be so large that they almost completely replace the gland. They are differentiated from ductal adenocarcinoma by their typically large size $(>10 \mathrm{~cm})$, honeycombed appearance, hypervascularity and central scar [19]. Serum tumour markers such as carcinoma embryonic antigen (CEA) and carbohydrate antigen (CA) 19.9 are typically within the normal range in cystic pancreatic neoplasms and elevation of these markers is also useful in distinguishing these lesions from ductal adenocarcinoma [18].

\section{Macrocystic serous adenoma}

Macrocystic serous adenomas are a variant of the microcystic adenoma. They are always benign and are characterised by unilocular or bilocular cysts that are greater than $2 \mathrm{~cm}$ in size. Similar to microcystic adenomas, there is a female predominance and the tumours more frequently occur in patients over 50 years of age. With large cystic spaces, these tumours can be difficult to distinguish from mucinous cystic neoplasms [21]. Generally, endoscopic ultrasound evaluation and aspiration are required to

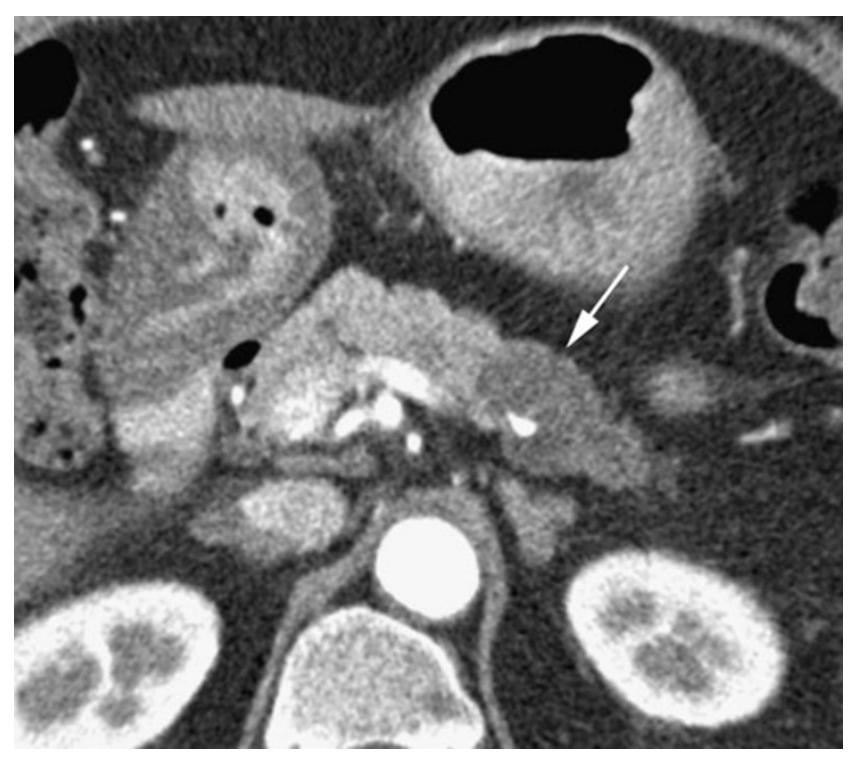

Fig. 4 Cystic lesion (arrow) in a 71-year-old woman with calcification and fine septations. Shown to be a microcystic cystadenoma 

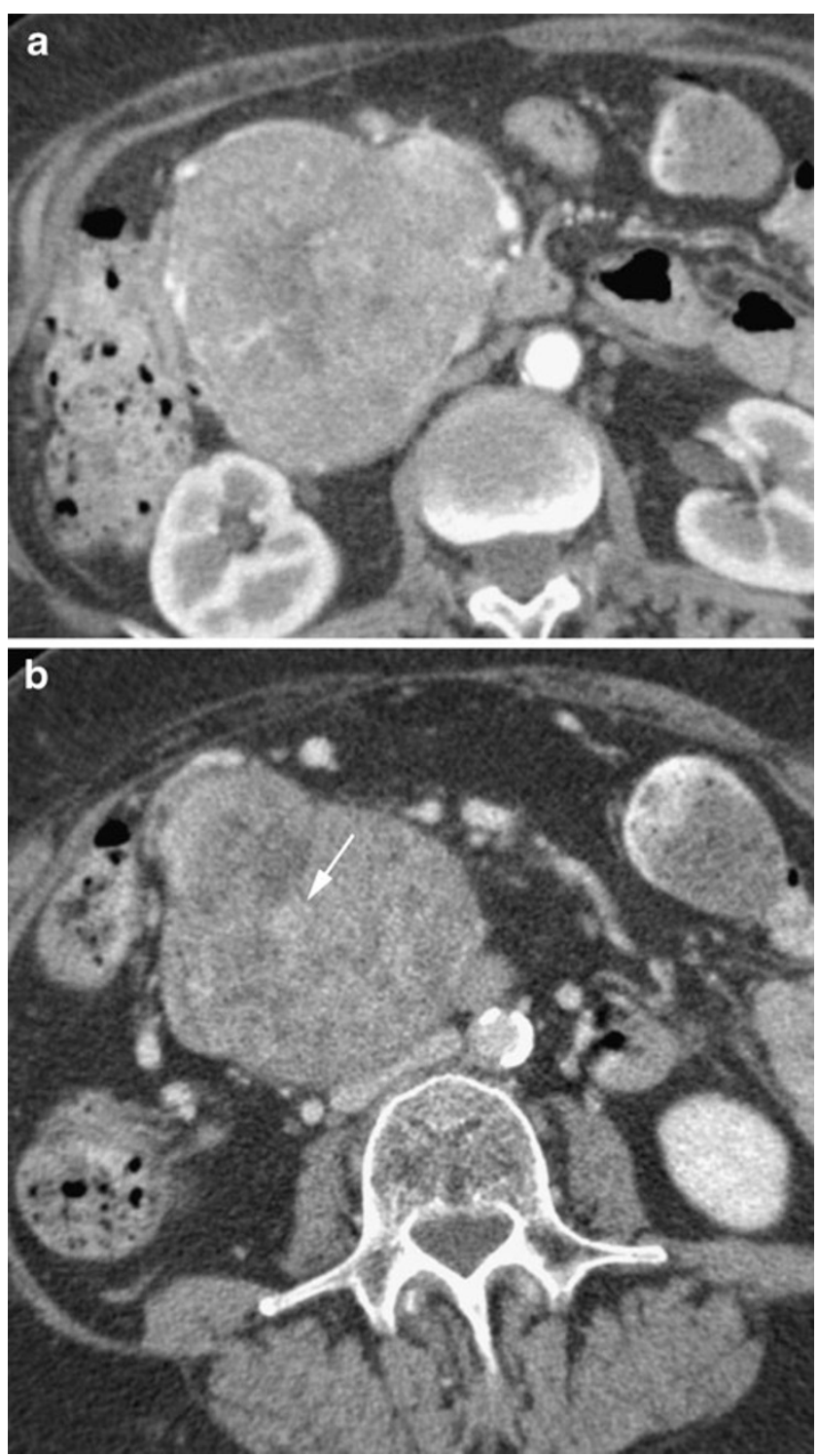

Fig. 5 a Arterial phase CT in a 67-year-old woman with a heterogeneous soft tissue mass shown to be a microcystic cystadenoma. It almost appears solid with some central fluid attenuation elements. Note it displaces vessels at the periphery rather than invading and obstructing. b Portal venous $\mathrm{CT}$ in the same patient. Note eccentric central enhancing 'scar' (arrow)

demonstrate a benign cytology and serous fluid rather than a mucinous aspirate.

Serous cystic neoplasms, in particular microcystic neoplasms, may present with symptoms of mass effect when large. Complications include obstruction of the second part of the duodenum and jaundice due to common bile duct obstruction. In these cases, complete tumour resection is the aim of management [18]. For asymptomatic lesions, management is more controversial. Tumours greater than $4 \mathrm{~cm}$ in size are thought more likely to grow and eventually cause symptoms and this may be used as a criterion for prophylactic resection [18]. Ultimately, this decision would involve a risk-benefit analysis of the patient's age and medical comorbidities, as well as tumour size and location in the pancreas.

\section{Mucinous cystic neoplasm}

Mucinous cystic neoplasms are the most common cystic neoplasm and occur almost exclusively in middle-aged women, (the 'mother' tumour) [22]. These tumours are potentially malignant (mucinous cystadenocarcinoma) and surgical resection is therefore the mainstay of treatment [18]. Most are incidental findings with the remainder presenting with non-specific abdominal symptoms including weight loss in metastatic cases.

These lesions tend to occur in the body or tail of the pancreas and appear as a unilocular or multilocular cyst, round or lobular with a smooth external margin (Fig. 6) [18, 23]. Mucinous cystic neoplasms tend to be large and
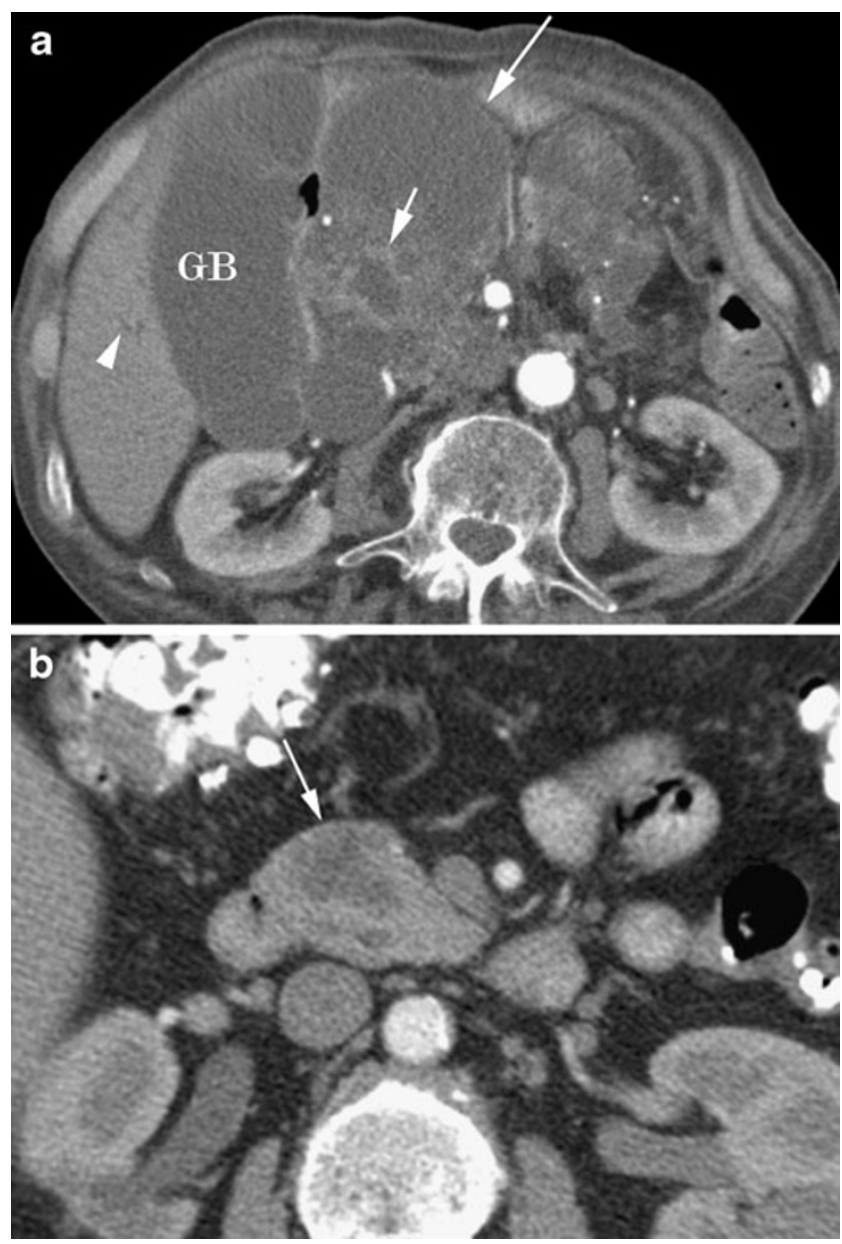

Fig. 6 a Large macrocystic cystadenocarcinoma (long arrow) in an 80-year-old man. Note the irregular soft tissue septations (short arrow). Mass causes biliary obstruction with a distended gallbladder $(G B)$ and intrahepatic ducts (arrowhead). b Axial arterial phase CT of a hypodense cystic mass (arrow) in the anterior pancreatic head in a 66 year old female shown to be a macrocystic cystadenoma 
peripheral calcification is present in approximately $16 \%$ of cases $[22,23]$, often with nodularity of the wall or septae with papillary projections into the lumen [18]. The internal Hounsfield density measurement depends on the proportion of mucoid content and haemorrhagic fluid [23].

Compared with the serous cystic neoplasms, cyst fluid CEA is elevated in mucinous cystic tumours and the degree of elevation correlates with the diagnosis of mucinous cystadenocarcinoma or mucinous cystadenoma with malignant potential [18]. Serum tumour markers CA 125 and CA 724 are also suggestive of a malignant or pre-malignant lesion [18].

\section{Intraductal papillary mucinous neoplasm}

Intraductal papillary mucinous neoplasm (IPMN) is a lowgrade malignancy that more frequently occurs in elderly patients with a male predominance (the 'grandfather' tumour). The tumour arises from a proliferation of the pancreatic ductal epithelium and may be predominantly cystic or papillary in composition. There is resultant excess mucin production and progressive dilatation of the main pancreatic duct or cystic dilatation of the branch ducts. These lesions frequently present with pancreatitis, abdominal pain or jaundice [24].

The presence of multifocal pancreatic cysts, present in $72 \%$ of cases, supports the diagnosis of IPMN as multifocality is unusual in other cystic neoplasms [25]. The key radiological feature is demonstration of communication between the cystic lesion and the pancreatic duct (Fig. 7) [26]. This is seen in only $18 \%$ on CT compared with $73 \%$ using MRI [25]. Additionally, MRI has proved superior at differentiating IPMN from other cystic tumours [27]. Bulging of the duodenal papilla into the lumen of the duodenum is also virtually diagnostic of IPMN [28]. The pancreatic parenchyma tends to be atrophic from mucinous obstruction. The tumour is usually not seen itself, but its presence is inferred from the sequelae of its pronounced mucin production (Fig. 8).
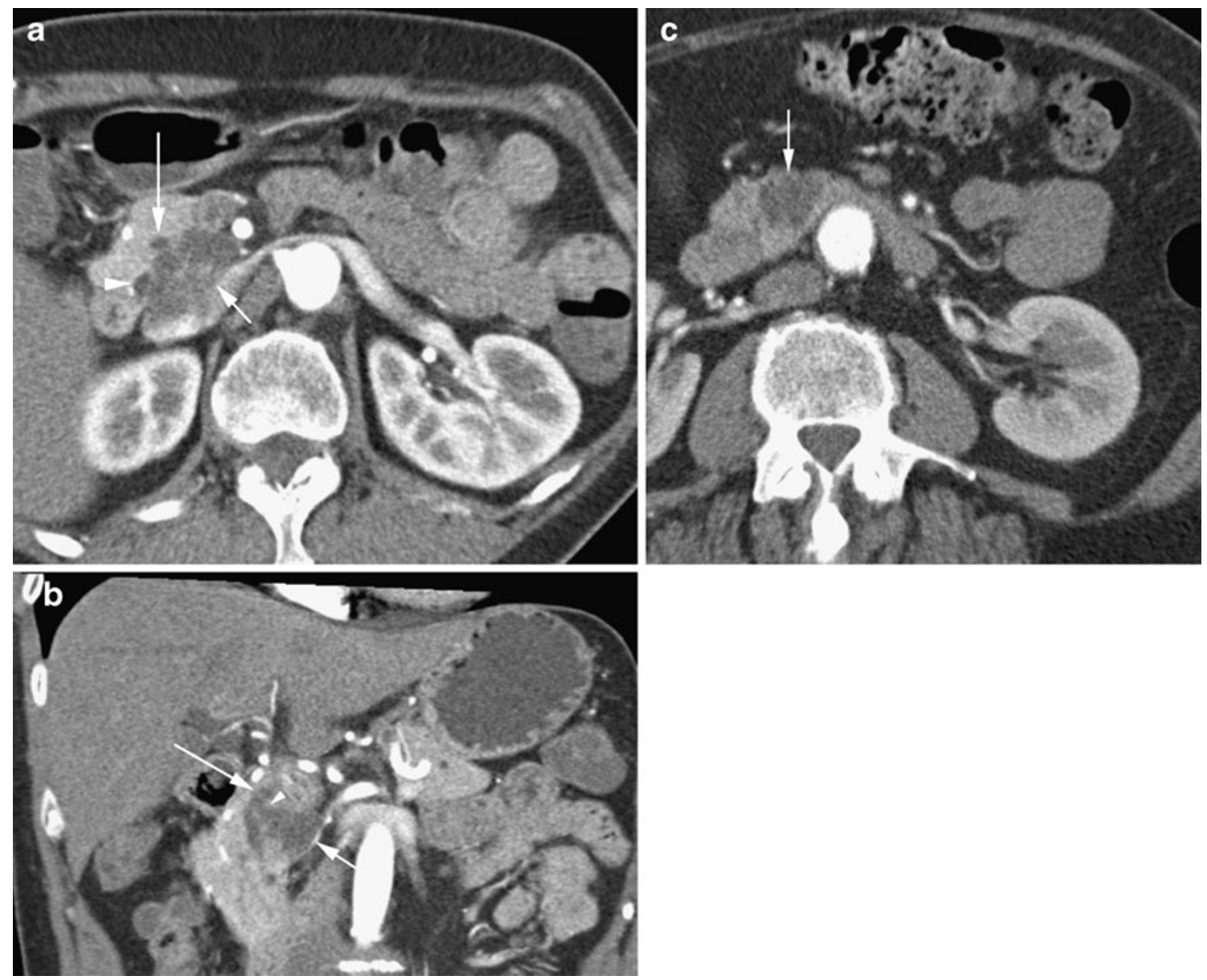

Fig. 7 a Arterial phase CT in a 59-year-old man showing a lobulated cystic mass (short arrow) shown to be a side branch IPMN. Note pancreatic duct (long arrow). b Coronal reformat in the same patient showing the lobulated IPMN (short arrow) extending to the pancreatic duct (long arrow) via a short side branch (arrowhead). c Axial CT showing a lobulated cystic side branch IPMN in a 66-year-old woman 


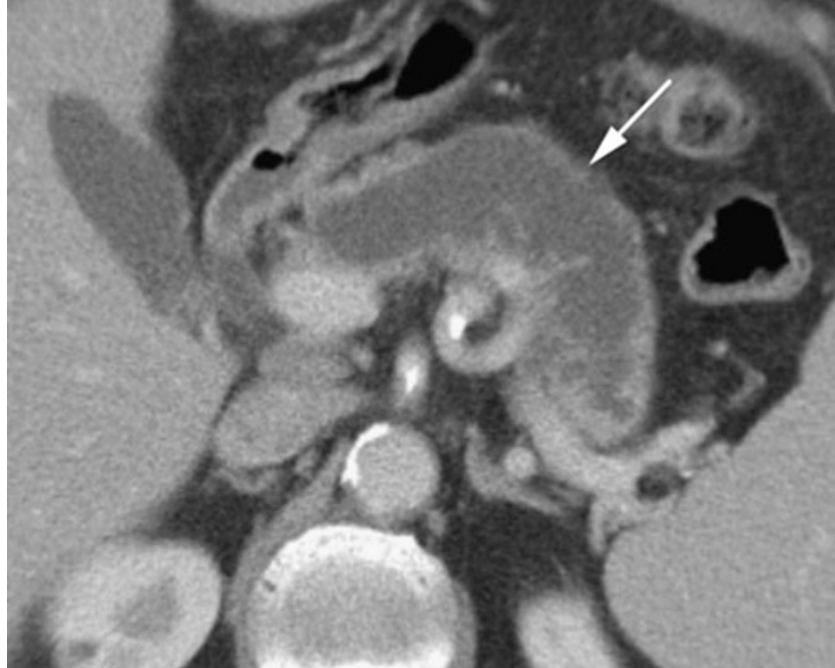

Fig. 8 Grossly distended main pancreatic duct (arrow) in an 83-yearold man with a main duct IPMN

The IPMN is classified on the basis of duct location as well as focal or diffuse disease:

1. Main duct type-moderate or marked dilatation of the main pancreatic duct

2. Segmental — can occur in the body or tail with upstream dilatation of the duct only

3. Branch duct type-most frequently in the uncinate process and appears as round or oval lobulated masses

4. Diffuse type-homogeneous low density appearance and differentiation from chronic pancreatitis can be difficult

Mural nodules help support the diagnosis.

Although frequently benign, they may contain carcinoma in situ or be frankly malignant with features of malignant degeneration including irregularity of the wall and thick septa with solid nodules [28]. Similar to mucinous cystic neoplasms, IPMN cyst fluid analysis shows high CEA and CA 72-4. A point of difference is high amylase in IPMN [18]. Elevated serum CEA and CA 125 are also associated with malignant lesions [18]. The best predictor of malignant potential, however, is tumour location, with the main duct type having a $70 \%$ chance of harbouring malignancy compared with $25 \%$ for the branch duct type [29]. All main duct type IPMN are recommended for resection, whereas branch duct type IPMN less than $3 \mathrm{~cm}$ in size and lacking mural nodules could potentially be managed conservatively [18].

\section{Solid pseudopapillary neoplasm (SPPN)}

Also sometimes referred to as solid pseudopapillary tumours (SPT), solid and papillary epithelial neoplasms
(SPEN), or Frantz's tumours, these tumours occur in young women in the 2nd and 3rd decades of life (the 'daughter' tumour), and are of fairly low malignant potential [30]. Metastases or vascular invasion may complicate $10-15 \%$ of cases; however, long-term survival is common in these patients following surgery and chemotherapy [31]. Complete surgical excision is always recommended, not only because of malignant potential but also because of the considerable size to which these tumours can grow. SPPNs begin as solid masses with a delicate internal vasculature. As the lesion enlarges, haemorrhagic and cystic degenera-
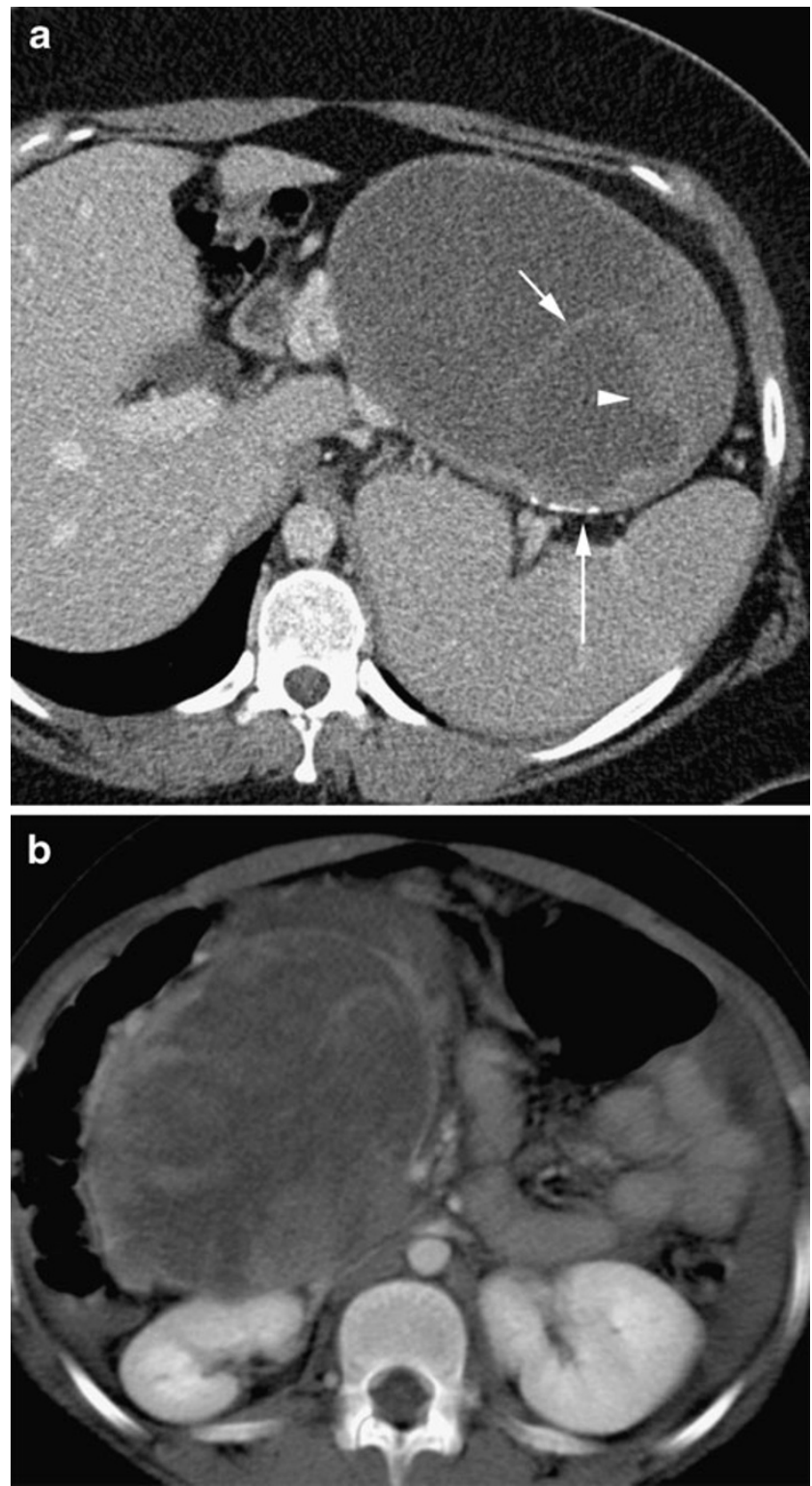

Fig. 9 a Portal venous phase CT with a solid and papillary tumour replacing the pancreatic tail in a 27-year-old woman. Note internal septations (short arrow), solid elements (arrow head) and peripheral calcification (long arrow). b Portal venous CT of a large heterogenous mass replacing the pancreatic head in a 19 year-old woman. Note the predominantly cystic density with irregular internal enhancing septations 
tion occurs, with only the cells close to the small vessels remaining intact [32].

The typical appearance is of a large, mixed solid and cystic lesion that occurs most commonly in the head or tail of the pancreas [33]. SPPNs tend to be well-encapsulated and contain haemorrhagic components, with more solid enhancing areas at the periphery and enhancement of the cyst wall [34]. Internal fluid-debris levels may be present and peripheral calcification can occur (Fig. 9) [32]. Despite their large size, they typically produce only minor dilatation of the pancreatic duct and no biliary distension [35]. Invasion of surrounding structures is also rare.

\section{Neuroendocrine tumours}

Neuroendocrine tumours, or islet cell tumours, account for $1-5 \%$ of all pancreatic neoplasms and arise from the endocrine pancreas [36]. Patients are typically in their 50s, with a slight male predominance. Most are solitary lesions that arise sporadically, but neuroendocrine tumours may also occur as part of genetic syndromes, such as multiple endocrine neoplasia type 1 (MEN 1), von HippelLindau, neurofibromatosis type 1 and tuberous sclerosis. Neoplasms in these conditions are often multiple, particularly in MEN 1.

Islet cell tumours are also classified as functional and non-functional, depending on whether the cells actively secrete hormones. The functional lesions present with classic clinical syndromes, depending on the cell type, and tend to be less than $3 \mathrm{~cm}$ in size at diagnosis because of systemic symptoms leading to presentation, while nonfunctioning tumours are more likely to present with symptoms of mass effect [37, 38]. Functional tumours include insulinomas (present with hypoglycaemia) and gastrinomas (present with peptic ulcer disease in Zollinger-Ellison Syndrome, and typically arise in the gastrinoma triangle bounded by the junction of the cystic
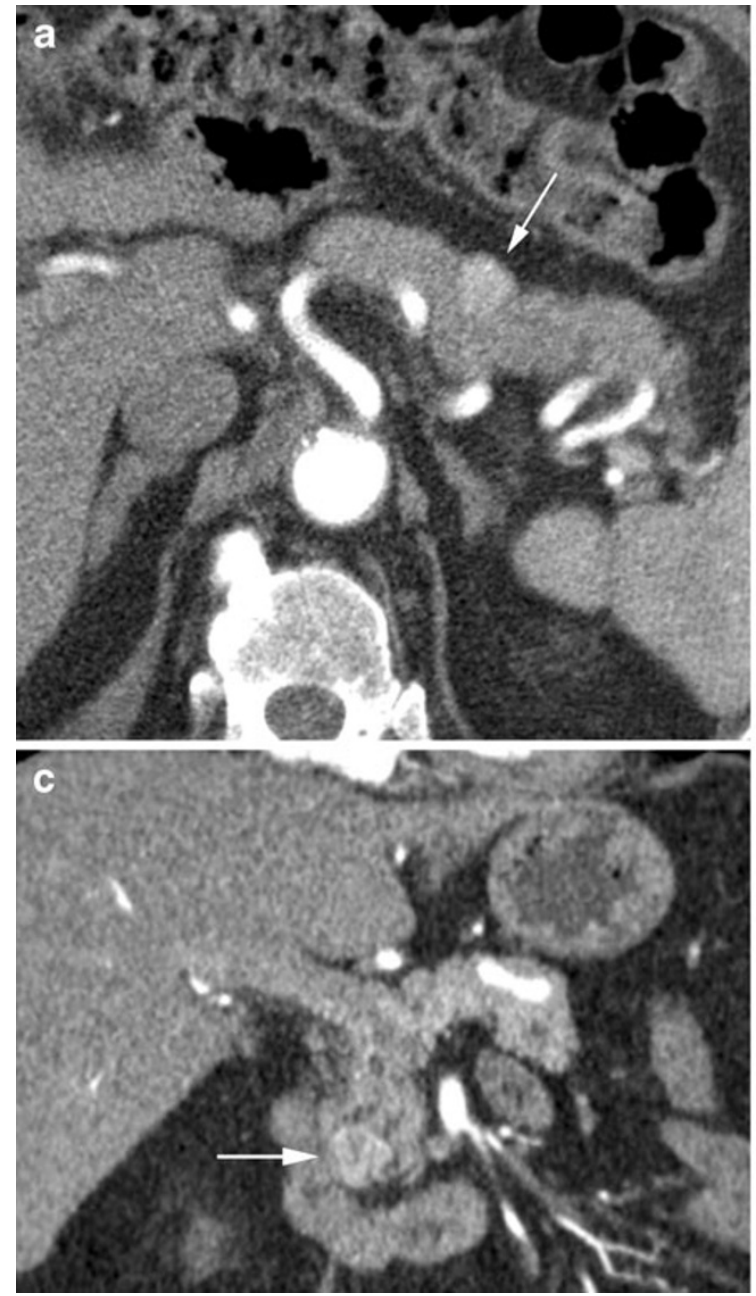

Fig. 10 a Arterially enhancing soft tissue insulinoma (arrow) in a 75year-old woman with hypoglycaemia. b, Axial arterial CT showing an arterially enhancing insulinoma in the uncinated process in a 76-year-

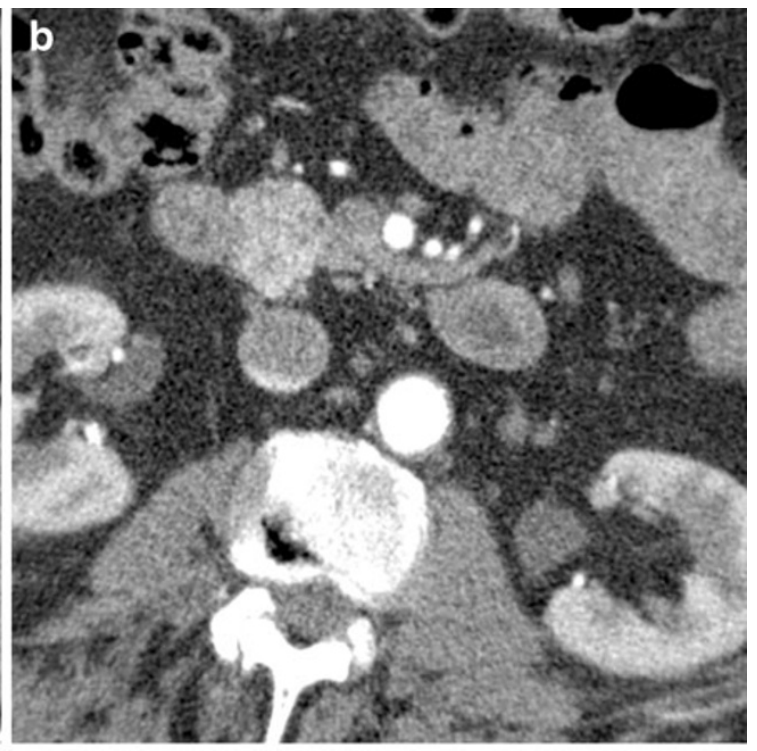

old man. c Coronal section in the same patient as figure 10b, showing the enhancing insulinoma (arrow) 
duct insertion to the common bile duct, the body of the pancreas, and the junction of the second and third portions of the duodenum), glucagonomas (present with diabetes, anaemia, weight loss and rash), VIPomas (presents with WDHA syndrome (watery diarrhoea, hypokalaemia, achlorhydria) and somatostatinomas (present with diabetes, cholelithiasis and steatorrhoea).

In general, islet cell tumours are hyperenhancing and may be within the gland or exophytic [39] (Fig. 10). These lesions lack the desmoplastic reaction seen in ductal adenocarcinoma and so rarely cause pancreatic duct dilatation or obstruction [36]. In a review of 1,483 pancreatic neuroendocrine tumours, 91\% were nonfunctioning, $2.5 \%$ were insulinomas, $4.2 \%$ gastrinomas, $1.6 \%$ glucagonomas and $0.9 \%$ VIPoma [37]. Overall, $60 \%$ were metastatic and $21 \%$ were locally advanced with no difference between functioning and non-functioning tumour groups. The most useful discriminator of malignant risk is tumour size with $90 \%$ of tumours under $20 \mathrm{~mm}$ being benign and $71 \%$ over $20 \mathrm{~mm}$ being malignant [40].

Neuroendocrine lesions do not characteristically show calcification and as lesions grow they develop cystic or necrotic areas. The periphery of the lesion will continue to arterially enhance [36]. Local vascular encasement or invasion, which is commonly seen with ductal adenocarcinoma, is rarely seen with neuroendocrine tumours [36]. Arterial phase imaging of the liver is vital to detect hepatic metastases.

\section{Pancreatic lymphoma}

Primary lymphoma of the pancreas is rare. Secondary involvement can occur via direct extension from the retroperitoneum. Symptoms are typical of those of systemic lymphoma and jaundice is rare, even with large masses at the pancreatic head.

Most pancreatic lymphomas are homogeneous, low attenuation masses. They tend to poorly enhance, but enhancement will also be homogeneous [41]. Morphological patterns are focal and diffuse. The focal type is distinguished from ductal adenocarcinoma by the lack of pancreatic duct dilatation. This tends to be mild and occurs late [41]. Lymphoma may encase vessels, but exerts little mass effect and unlike adenocarcinoma does not typically invade vessels or produce thrombosis (Fig. 11). Lymphadenopathy below the renal vein level is strongly supportive of a diagnosis of lymphoma [42]. The diffuse type can mimic acute pancreatitis with global gland enlargement; however, it should be clinically distinguishable. Calcification should not be present in primary pancreatic lymphoma and this may also be a key feature in diagnostic imaging [42].
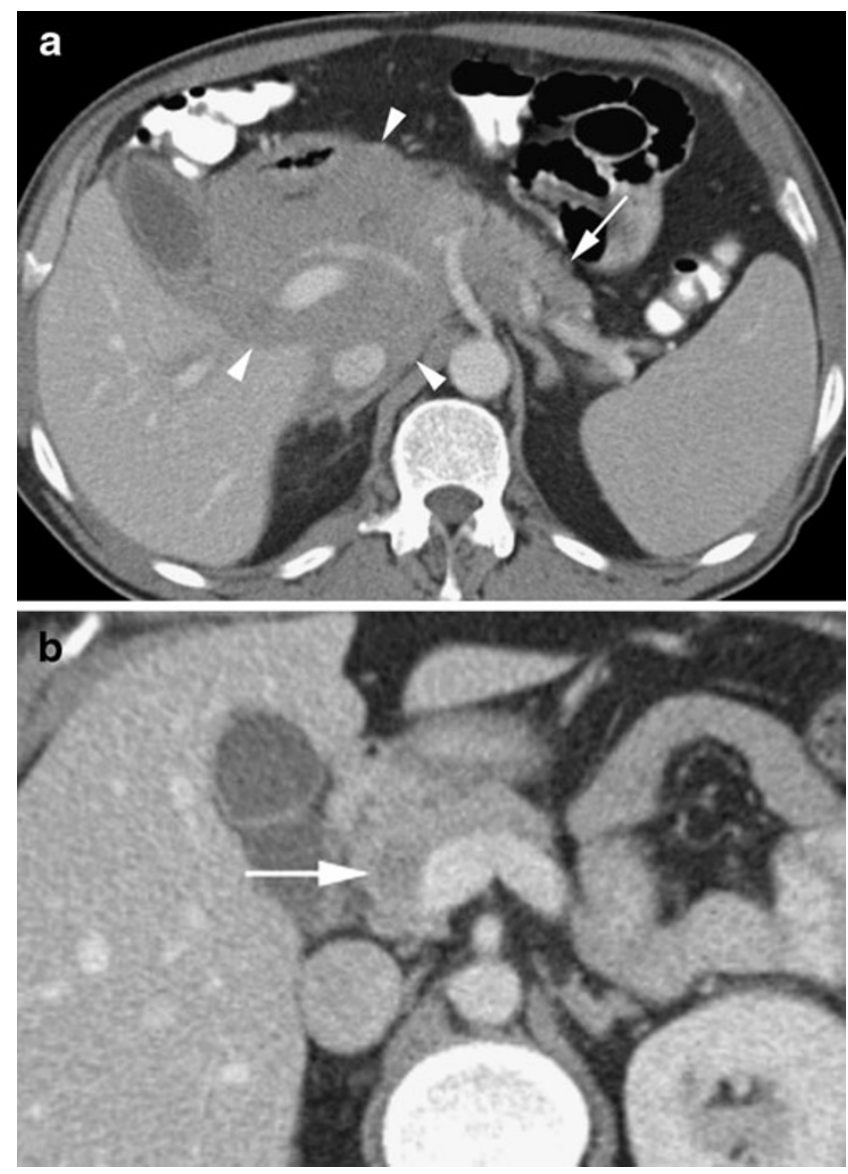

Fig. 11 a Homogeneous large lymphomatous soft tissue mass (arrowheads) encasing vessels in a 72-year-old man and replacing the head of the pancreas, with normal body and tail (arrow). b Subtle low density nodule (arrow) in the head of the pancreas in a 33-yearold with diffuse large B cell lymphoma resolved with treatment and was presumed lymphomatous involvement

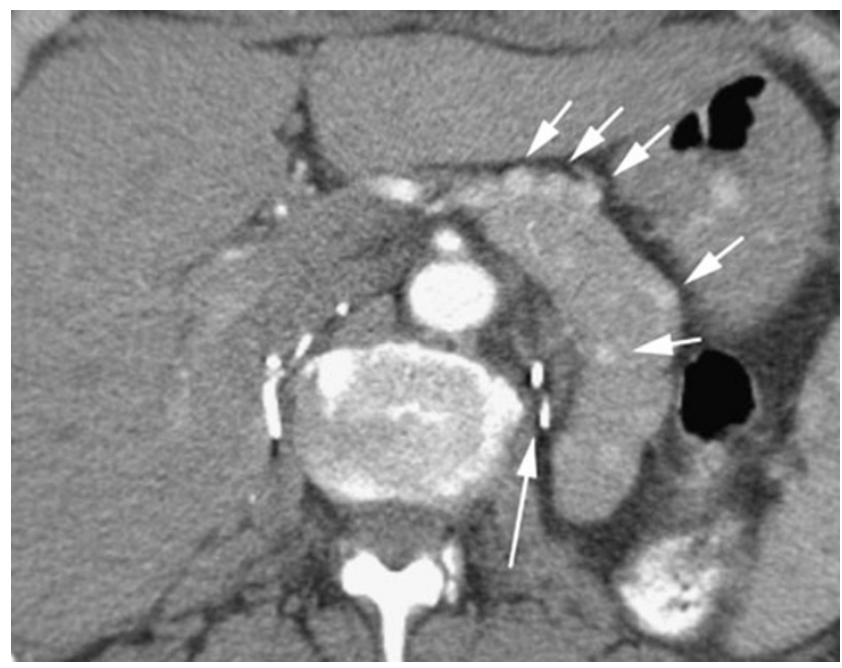

Fig. 12 Arterial phase CT in a 60-year-old woman showing innumerable hypervascular pancreatic renal cell carcinoma metastases (short arrows). Note nephrectomy clips (long arrow) 


\section{Metastases}

Metastases to the pancreas are rare, found in only $3-12 \%$ of patients at autopsy with advanced malignancy [43]. The most frequent primary lesions are bronchogenic carcinoma and renal cell carcinoma. Other documented sources are breast, thyroid, melanoma, gastrointestinal tract and hepatocellular carcinoma [43].

Patterns of metastatic deposits include a single focal mass (most common), diffuse gland enlargement and multiple pancreatic nodules (Fig. 12). Lesions are usually hypodense to pancreas and can show the enhancement pattern of the primary lesion. Both renal cell carcinoma and bronchogenic carcinoma tend to result in arterially enhancing metastases, while a peripheral rim of enhancement may be seen with other primaries [43]. The diffuse form tends to be isodense to pancreas post-contrast medium administration but may show low attenuation areas centrally [44].

\section{Conclusion}

As demonstrated, the spectrum of pancreatic neoplasms has varying age and gender profiles as well as clinical presentations. This information, coupled with knowledge of the typical CT characteristics, allows for reasonable confidence in diagnosis. Multiphase CT, with the range of post-processing techniques available, also plays a crucial role in determining the resectability of pancreatic ductal adenocarcinoma, the most common pancreatic neoplasm.

\section{References}

1. Winter TC, Ager JD, Nghiem HV et al (1996) Upper gastrointestinal tract and abdomen: water as an orally administered contrast agent for helical CT. Radiology 201:365-370

2. Sener SF, Fremgen A, Menck HR, Winchester DP (1999) Pancreatic cancer: a report of treatment and survival trends for 100,313 patients diagnosed from 1985-1995, using the National Cancer Database. J Am Col Surg 189(1):1-7

3. Klauss M, Alt CD, Welzel $T$ et al (2009) Multidectector CT evaluation of the course of nonresectable pancreatic carcinomas with neoadjuvant therapy. Pancreatology 9:621-630

4. Gangi S, Fletcher JG, Nathan MA et al (2004) Time interval between abnormalities seen on $\mathrm{CT}$ and the clinical diagnosis of pancreatic cancer: retrospective review of CT scans obtained before diagnosis. AJR Am J Roentgenol 182:897903

5. McNulty NJ, Francis IR, Cohan RH et al (2001) Multi-detector row helical CT of the pancreas: effect of contrast-enhanced multiphase imaging on enhancement of the pancreas, peripancreatic vasculature, and pancreatic adenocarcinoma. Radiology 220:97-102

6. Deshmukh SD, Willmann JK, Jeffrey RB (2010) Pathways of extrapancreatic perineural invasion by pancreatic adenocarcinoma: evaluation with $3 \mathrm{D}$ volume-rendered MDCT imaging. AJR Am J Roentgenol 194:668-674
7. Bluemke DA, Cameron JL, Hruban RH et al (1995) Potentially resectable pancreatic adenocarcinoma: spiral CT assessment with surgical and pathologic correlation. Radiology 197:381-385

8. Tabuchi T, Itoh K, Ohshio G (1999) Tumor staging of pancreatic adenocarcinoma using early- and late-phase helical CT. AJR Am J Roentgenol 173:375-380

9. Sai M, Mori H, Kiyonaga M et al (2010) Peripancreatic lymphatic invasion by pancreatic carcinoma: multi-detector row CT. Abdom Imaging 35(2):154-162

10. Horton KM, Fishman EK (2002) Multidetector CT angiography of pancreatic carcinoma: part 2, evaluation of venous involvement. AJR Am J Roentgenol 178:833-836

11. Horton KM, Fishman EK (2002) Multidetector CT angiography of pancreatic carcinoma: part 1, evaluation of arterial involvement. AJR Am J Roentgenol 178:827-831

12. Phoa SSKS, Reeders JWAJ, Stoker J et al (2000) CT criteria for venous invasion in patients with pancreatic head carcinoma. $\mathrm{Br} \mathrm{J}$ Radiol 73:1159-1164

13. Li H, Zeng MS, Zhou KR et al (2005) Pancreatic adenocarcinoma: the different ct criteria for peripancreatic major arterial and venous invasion. J Comput Assist Tomogr 29:170-175

14. Lu DSK, Reber HA, Krasny RM et al (1997) Local staging of pancreatic cancer: criteria for unresectability of major vessels as revealed by pancreatic-phase, thin-section helical CT. AJR Am J Roentgenol 168:1439-1443

15. Bipat S, Phoa SSKA, van Delden OM et al (2005) Ultrasonography, computed tomography and magnetic resonance imaging for diagnosis and determining resectability of pancreatic adenocarcinoma: a meta-analysis. J Comput Assist Tomogr 29:438-445

16. Zamboni GA, Kruskal JB, Vollmer CM et al (2007) Pancreatic adenocarcinoma: value of multidetector $\mathrm{CT}$ angiography in preoperative evaluation. Radiology 245(3):770-778

17. Sahani DV, Shah ZK, Cataloano OF et al (2008) Radiology of pancreatic adenocarcinoma: current status of imaging. J Gastroenterol Hepatol 23(1):23-33

18. Ng DZW, Goh BKP, Tham EHW et al (2009) Cystic neoplasms of the pancreas: current diagnostic modalities and management. Ann Acad Med Singapore 38:251-259

19. Buck JL (1990) Hayes DO (1983) Microcystic adenoma of the pancreas. Radiographics 10:313-322

20. Friedman AC, Lichtenstein JE, Dachman AH (1983) Cystic neoplasms of the pancreas. Radiology 149:45-50

21. Khurana B, Mortele KJ, Glickman J et al (2003) Macrocystic serous adenoma of the pancreas; radiologic-pathologic correlation. AJR Am J Roentgenol 181:119-123

22. De Lima JE, Javitt MC, Mathur SC (1999) Mucinous cystic neoplasm of the pancreas. Radiographics 19:807-811

23. Buetow PC, Rao P, Thompson LDR (1998) Mucinous cystic neoplasms of the pancreas: radiologic-pathologic correlation. Radiographics 18:433-449

24. Baiocchi GL, Portolani N, Missale G et al (2010) Intraductal papillary mucinous neoplasm of the pancreas (IPMN): clinicopathological correlations and surgical indications. World J Surg Oncol 8:25

25. Waters JA, Schmidt CM, Pinchot JW et al (2008) CT vs MRCP: optimal classification of IPMN type and extent. J Gastrointest Surg 12:1010-1109

26. Lim JH, Lee G, Oh YL (2001) Radiologic spectrum of intraductal papillary mucinous tumour of the pancreas. Radiographics $21: 323-340$

27. Song SJ, Lee JM, Kim YJ et al (2007) Differentiation of intraductal papillary mucinous neoplasms from other pancreatic cystic masses: comparison of multirow-detector CT and MRI imaging using ROC analysis. J Magn Reson Imaging 26(1):8693 
28. Procacci C, Megibow AJ, Carbognin G et al (1999) Intraductal papillary mucinous tumour of the pancreas: a pictorial essay. Radiographics 19:1447-1463

29. Tanaka M, Chari S, Adsay V et al (2006) International consensus guidelines for management of intraductal papillary mucinous neoplasms and mucinous cystic neoplasms of the pancreas. Pancreatology 6:17-32

30. Patil TB, Shrikhande V, Kanhere A et al (2006) Solid pseudopapillary neoplasm of the pancreas: a single institution experience of 14 cases. HPB 8:148-150

31. Sperti C, Berselli M, Pasquali C et al (2008) Aggressive behaviours of solid-pseudopapillary tumor of the pancreas in adults: a case report and review of the literature. World $\mathrm{J}$ Gastroenterol 14(6):960-965

32. Kamat RN, Naik LD, Joshi RM et al (2008) Solid pseudopapillary tumour of the pancreas. Indian J Pathol Microbiol 51:271-273

33. Coleman KM, Doherty MC, Bigler SA (2003) Solidpseudopapillary tumor of the pancreas. Radiographics 23:16441648

34. Choi JY, Kim MJ, Kim SH et al (2006) Solid pseudopapillary tumor of the pancreas: typical and atypical manifestations. AJR Am J Roentgenol 187:178-186

35. Dong DJ, Zhang SZ (2006) Solid-pseudopapillary tumor of the pancreas: CT and MRI features of 3 cases. Hepatobiliary Pancreat Dis Int 5:300-304
36. Lee DS, Jeffrey RB, Kamaya A (2009) Islet-cell tumors of the pancreas: spectrum of MDCT findings: a pictorial essay. Appl Radiol 38:10-28

37. Halfdanarson TR, Rabe KG, Rubin J, Petersen GM (2008) Pancreatic neuroendocrine tumors (PNETS): incidence, prognosis and recent trend toward improved survival. Ann Oncol 19:1727-1733

38. Kazanjian KK, Reber HA, Hines OJ (2006) Resection of pancreatic neuroendocrine tumors: results of 70 cases. Arch Surg 141:765-770

39. Horton KM, Hruban RH, Yeo C (2006) Multi-detector row CT of pancreatic islet cell tumors. Radiographics 26:453-464

40. Schindl M, Kaczirek K, Kaserer K, Niederle B (2000) Is the new classification of neuroendocrine pancreatic tumors of clinical help? World J Surg 24:1312-1318

41. Merkle EM, Bender GN, Brambs HJ (2000) Imaging findings in pancreatic lymphoma: differential aspects. AJR Am J Roentgenol 174:671-675

42. Prayer L, Schurawitzki H, Malleck R, Mostbeck G (1992) CT in pancreatic involvement of non-Hodgkin lymphoma. Acta Radiol 33:123-127

43. Scartarige JC, Horton KM, Sheth S et al (2001) Pancreatic parenchymal metastases: observation on helical CT. AJR Am J Roentgenol 176:695-699

44. Merkle EM, Boaz T, Kolokythas O et al (1998) Metastases to the pancreas. Br J Radiol 71:1208-1214 